Pacific Journal of Mathematic 


\section{THE $H_{p}$-PROBLEM AND THE STRUCTURE OF $H_{p}$-GROUPS}

D. R. Hughes AND J. G. Thompson

1. Introduction. Let $G$ be a group, $p$ a prime, and $H_{p}(G)$ the subgroup of $G$ generated by the elements of $G$ which do not have order $p$. In a research problem in the Bulletin of the American Mathematical Society, one of the authors posed the following problem: is it always true that $H_{p}(G)=1, H_{p}(G)=G$, or $\left[G: H_{p}(G)\right]=p$ ? This problem is easily settled in the affirmative for $p=2$, and a similar answer was recently given for $p=3$ ([5]). In this paper (Section 2) we give an affirmative answer for the case that $G$ is finite and not a $p$-group. Furthermore (Section 3) we are able to give a rather precise description of the structure of $G$ in the most interesting case, when $\left[G: H_{p}(G)\right]=p$. This structure theorem depends heavily on the deep results of Hall and Higman ([4]) and Thompson ([6]) on finite groups. If $H(\neq 1)$ is a finite group and there exists a group $G$ such that $H_{p}(G)$ is isomorphic to $H$, where $H_{p}(G) \neq G$, then we call $H$ an $H_{p}$-group; it is seen that $H_{p}$-groups are natural generalizations of "Frobenius groups." By a Frobenius group we mean a finite group $G$ possessing an automorphism $\sigma$ of prime order $p$ such that $x^{\sigma}=x$ if and only if $x=1$. It is easy to show that this implies

$$
x^{1+\sigma+\cdots+\sigma^{p-1}}=x\left(x^{\sigma}\right) \cdots\left(x^{\sigma^{p-1}}\right)=1,
$$

for all $x$ in $G$. This last equation characterizes $H_{p}$-groups, ${ }^{1}$ and as a generalization of Thompson's result ([6]) that Frobenius groups are nilpotent, we show that $H_{p}$-groups are solvable, among other things.

Throughout the paper, if $B$ is a group, $A$ a subgroup of $B$, then $N_{B}(A)$ and $C_{B}(A)$ mean, respectively, the normalizer and centralizer of $A$ in $B$. By $Z(A)$ we mean the center of $A$.

2. The $H_{p}$-problem. Let $G$ be a group, and let $H=H_{p}(G)$. Suppose

(1) $G$ is finite,

(2) $G$ is not a $p$-group,

(3) the index of $H$ in $G$ is greater than $p$,

(4) $G$ is a group of minimal order satisfying (1), (2), (3). Note that every element of $G$ which is not in $H$ has order $p$.

Let $q$ be a prime dividing $[G: 1], q \neq p$, and let $Q$ be a Sylow $q$ group of $G$; then $Q$ is also a Sylow $q$-group of $H$. Let $N=N_{G}(Q)$; then

Received January 16, 1959. The first author was supported in part by the United States Air Force through the Air Force Office of Scientific Research of the Air Research and Development Command under contract No. AF 18 (600)-1383.

1 Unless the group is a $p$-group; see Theorem 2. 
by the Frattini argument (see [1], p. 117, for instance), $G=N H$. Thus $[G: 1]=[N H: 1]=[N: 1][H: 1] /[N \cap H: 1]$.

First, let us suppose $N \neq G$. Then clearly $H_{p}(N) \subseteq H_{p}(G)$, so $H_{p}(N) \subseteq H \cap N$. Since $Q \subseteq H_{p}(N)$, it follows that $H_{p}(N) \neq 1$, so $\left[N: H_{p}(N)\right] \leq p$, and hence $[N: N \cap H] \leq p$. So $p^{2}=[G: H]=$ $[G: 1] /[H: 1]=[N: 1] /[N \cap H: 1]=[N: N \cap H] \leq p$. This is impossible, so we must have $N=G$, and thus $Q$ is normal in $G$.

Now let $Q_{1}(\neq 1)$ be any subgroup of $Q$, normal in $G$, and consider $G / Q_{1}$. Clearly $H_{p}\left(G / Q_{1}\right)=1$ or $H_{p}\left(G / Q_{1}\right)$ has index $p$ in $G / Q_{1}$, unless $G / Q_{1}$ is a $p$-group. Indeed, it is obvious that $H_{p}\left(G / Q_{1}\right) \subseteq H / Q_{1}$. But $\left[G / Q_{1}: H / Q_{1}\right]=[G: H]=p^{2}$, so $\left[G / Q_{1}: H_{p}\left(G / Q_{1}\right)\right] \geq\left[G / Q: H / Q_{1}\right]=p_{2}$ implies $H_{p}\left(G / Q_{1}\right)=1$. So $G / Q_{1}$ is a $p$-group.

LEMMA 1. If $[G: H]=p^{2}$, then $Q$ is an elementary abelian $q$-group, none of whose proper subgroups $(\neq 1)$ is normal in $G, Q$ is normal in $G$, and $G=P Q$, where $P$ is a Sylow p-group of $G$.

Proof. We have shown that $Q$ is normal. If $Q_{1}$ above is taken to be the Frattini subgroup of $Q$, then $Q_{1}$ is normal in $G$, since it is characteristic in $Q$. Since $Q_{1} \neq Q, G / Q_{1}$ cannot be a $p$-group, so we must have $Q_{1}=1$. Thus $Q$ is elementary abelian. Since $G / Q$ is a $p$-group, it is clear that $G=P Q$, and the rest of the lemma follows similarly.

In what follows, $P$ is a Sylow $p$-group of $G$ and $P_{0} \subseteq P$ is a Sylow $p$-group of $H$; clearly $\left[P: P_{0}\right]=p^{2}$ and $P_{0}$ is normal in $P$, since $P_{0}=P \cap H$.

If $x(\neq 1)$ is in $Q$, while $a$ is in $G$, not in $H$, and if $a x=x a$, then $a x$ has order $p q$. But $a x$ is not in $H$, since $a$ is not in $H$, and thus $a x$ has order $p$; hence $a x \neq x a$. If $P_{0}=1$, then $P$, of order $p^{2}$, is an automorphism group of $H=Q$ such that no non-identity element of $P$ fixes any non-identity element of $Q$. But by ([2], pp. 334-335) this means that $P$ is cyclic, whereas $P$ is clearly elementary abelian in this case (for all its elements have order $p$ ). So $P_{0} \neq 1$.

Since $P_{0}$ is normal in $P, P_{0} \cap Z(P) \neq 1$ (see [3], p. 35, for instance). Let $z$ be an element of $P_{0} \cap Z(P)$, chosen to have order $p$, and let $Z_{0}$ be the subgroup (of order $p$ ) generated by $z$; note that $z$ and $Z_{0}$ are contained in $H$. Let $K=Z_{0} Q$, and observe that $[K: 1]=p[Q: 1]$. Let $a$ be an element of $G$, not in $H$, and $G_{1}=\{a, K\}=$ the group generated by $a$ and $K$. Then $Q \subseteq H_{p}\left(G_{1}\right) \subseteq H \cap G_{1} \neq G_{1}$, so $\left[G_{1}: H_{p}\left(G_{1}\right)\right]=p$, by induction. Hence $Z_{0} \subseteq K \subseteq H_{p}\left(G_{1}\right)$, so there must be an element $y$ in $K$ of order $p q$. Then $y^{p}$ is in $Q$ and $y^{q}$ is in $x^{-1} Z_{0} x$, for some $x$ in $K$, since $Z_{0}$ is a Sylow $p$-group of $K$. By adjusting our choice of $P$, we can assume that $y^{q}$ is in $Z_{0}$; let $u=y^{p}, v=y^{q}$. Then $u \neq 1, v \neq 1, u$ is in $Q, v$ is in $Z_{0}$, and $u v=v u$. So if $Q_{1}=\{u\}$, we have $Z_{0} \subseteq C_{G}\left(Q_{1}\right)$. But then $x^{-1} Z_{0} x \subseteq C_{G}\left(x^{-1} Q_{1} x\right)$, and if $x$ is in $P$, this implies $Z_{0} \subseteq C_{G}\left(x^{-1} Q_{1} x\right)$, for all $x$ in $P$. But, from Lemma 1 , the subgroup generated by all 
$x^{-1} Q_{1} x$, as $x$ ranges over $P$, must be $Q$, and so $Z_{0} \subseteq C_{G}(Q)$. Since $Z_{0}$ is in the center of $P$, it follows that $Z_{0}$ is normal in $G$, so we consider $G / Z_{0}$. One easily sees that $H_{p}\left(G / Z_{0}\right) \subseteq H / Z_{0}$, and $H_{p}\left(G / Z_{0}\right)$ equals neither 1 nor $G / Z_{0}$. Hence $p^{2}=[G: H]=\left[G\left|Z_{0}: H\right| Z_{0}\right] \leq\left[G \mid Z_{0}: H_{p}\left(G \mid Z_{0}\right)\right]=p$, which is a contradiction. So:

THEOREM 1. If $H_{p}(G) \neq 1$ or $G$, and if $G$ is finite and not a $p$ group, then $\left[G: H_{p}(G)\right]=p$.

If $G$ is a $p$-group, or is infinite, the situation seems more inaccessible; as remarked earlier, Theorem 1 still holds if $p=2$ or 3 , no matter what $G$ is. But the proof for $p=3$ (see [5]) utilizes the Burnside theorem (for $p=3$ ) and this strongly suggests that the infinite case at least is considerably harder.

3. Structure of $H_{p}$-groups. Let us suppose that $G$ is a finite group, and that $H=H_{p}(G)$ has index $p$ in $G$. Then we say that $H$ is an $H_{p}$-group.

Theorem 2. If $H$ is not a p-group, then $H$ is an $H_{p}$-group if and only if $H$ has an automorphism $\sigma$ of order $p$ such that

$$
x^{1+\sigma+\cdots+\sigma^{p-1}}=1 \text {, }
$$

for all $x$ in $H$.

Proof. If $H=H_{p}(G)$, let $a$ be in $G, a$ not in $H$, and define $x^{\sigma}=$ $a^{-1} x a$, for $x$ in $H$. Since $(a x)^{p}=1$, while $(a x)^{p}=a^{p}(x)\left(x^{\sigma}\right) \cdots\left(x^{\sigma^{p-1}}\right)$, the equation of the theorem follows immediately.

Conversely, if $\sigma$ exists satisfying the hypotheses of the theorem, then let $G$ be the holomorph of $H$ by the automorphism group $\{\sigma\}$. It is easy to see that $H_{p}(G) \subseteq H$. Since $H_{p}(G) \neq 1$ (for $H$ is not a $p$-group), it follows that $\left[G: H_{p}(G)\right]=p$, from Theorem 1 , so $H_{p}(G)=H$.

Note that if $x^{\sigma}=x$, then the equation of Theorem 2 implies $x^{p}=1$. So if $p$ does not divide the order of the $H_{p}$-group $H$, then $H$ is even a Frobenius group, and so is nilpotent ([6]).

THEOREM 3. If $H$ is an $H_{p}$-group, then $H=P K$, where $P$ is a Sylow p-group of $H, K$ is normal in $H$ and is nilpotent, and $P \cap K=1$. In particular, $H$ is solvable.

Proof. We can assume that $P \neq 1$, and that $H$ is not a $p$-group. Inductively, suppose the theorem is true for all $H_{p}$-groups whose order is less than the order of $H$, and (using Theorem 2) let $\gamma$ be an automorphism of $H$, of order $p$, such that

$$
x^{1+\gamma+\cdots+\gamma^{p-1}}=1 \text {, all } x \text { in } H .
$$


If $A$ is a $\gamma$-invariant subgroup of $H$, then $A$ is an $H_{p}$-group or is a $p$ group, while if $B$ is a $\gamma$-invariant normal subgroup of $H$, then $H / B$ is an $H_{p}$-group or is a $p$-group.

Now let $B$ be any $\gamma$-invariant subgroup of $P, B$ normal in $P, B \neq 1$; let $N=N_{H}(B)$. If $N=H$, then $H / B$ is an $H_{p}$-group, so $H / B=(P / B)\left(K_{1} / B\right)$, where $K_{1} / B$ is normal in $H / B$ and is nilpotent. So $K_{1}$ is normal in $H$ and since $K_{1} / B$ is $\gamma$-invariant in $H / B$, so is $K_{1} \gamma$-invariant in $H$. So $K_{1}$ is an $H_{p}$-group. If $K_{1} \neq H$, then $K_{1}=B K$, where $K$ is normal in $K_{1}$ and is nilpotent, and $K \cap B=1$. But then $K$ is characteristic in $K_{1}$, hence is normal in $H$; every Sylow $q$-group of $H, q \neq p$, is in $K$. So $K$ is characteristic in $H$ and clearly $H=P K, P \cap K=1$.

If $K_{1}=H$ for every such $B$, then $B=P$ is the only $\gamma$-invariant normal subgroup of $P$, other than 1 . Hence in particular $P$ is elementary abelian. Then $H / P$ is an $H_{p}$-group, and even a Frobenius group, so is nilpotent. Furthermore (since $H$ is then solvable), $H=P K$, where $K$ is isomorphic to $H / P$. Let $K=Q_{1} Q_{2} \cdots Q_{t}$, where $Q_{i}$ is a Sylow $q_{i}$-group of $K$ (and of $H$ ) for distinct primes $q_{1}, q_{2}, \cdots, q_{t}$.

Now let $G$ be the holomorph of $H$ with the group $\{\gamma\}$. Then, by the Frattini argument, $N_{G}\left(Q_{i}\right) \cap H \neq N_{G}\left(Q_{i}\right)$, so by an appropriate choice of $\gamma_{i}$ in $G, \gamma_{i}$ not in $H$, we can assume that $Q_{i}$ is $\gamma_{i}$-invariant. Thus $P Q_{i}$ is $\gamma_{i}$-invariant and so it is an $H_{p}$-group (it is straightforward to check that any element of $G$, not in $H$, can play the role of $\gamma$ ). ${ }^{2}$

If $t>1$, then $P Q_{i}$ has order smaller than $H$, so $Q_{i}$ is normal in $P Q_{i}$. Thus both $P$ and $K$ are contained in $N_{H}\left(Q_{i}\right)$, so $Q_{i}$ is normal in $H$, hence $K$, which is the direct product of the $Q_{i}$, is normal in $H$, so we are done.

If $t=1$, let $Q=Q_{1}$, and as above, choose $\gamma$ in $G$, not in $H$, so that $Q$ is $\gamma$-invariant. If $Q_{0} \neq 1$ is a $\gamma$-invariant normal subgroup of $Q$, then $P Q_{0}$ is an $H_{p}$-group, smaller than $H=P Q$ if $Q_{0} \neq Q$; thus $P$ normalizes $Q_{0}$, so $Q_{0}$ is normal in $H$. Then by considering $H / Q_{0}$, we find that $Q / Q_{0}$ is normal, so $Q$ is normal in $H$, and again we are done. Thus we can assume that $Q$ is elementary abelian with only trivial $\gamma$-invariant normal subgroups.

Now we consider the holomorph $G$ again. The maximal normal $p$ group of $G$ is $P$, since $\{\gamma\}$ (as part of $G$ ) is not normalized modulo $P$ by $Q$. Then $G / P$ is a solvable (and in particular, $p$-solvable) group of automorphisms of the elementary abelian group $P$, and $G / P$ has no normal $p$-group $(\neq 1)$. Furthermore, this representation of $G / P$ as a linear transformation group on $P$ is faithful, since $C_{H}(P) \cap Q=1$ (otherwise $C_{H}(P) \cap Q$ would be a non-trivial $\gamma$-invariant normal subgroup of $Q$ ). Thus we can utilize Theorem B of Hall and Higman ([4]); since $Q$ is abelian, Theorem $\mathrm{B}$ asserts that $\gamma$, as a linear transformation of $P$, has the minimal

2 In these references to the holomorph $G$, we are not making a distinction between an element as an automorphism of $H$ and as an element of $G$; the automorphism is actually identified with an element of $G$ which induces the prescribed automorphism in $H$. 
polynomial $(x-1)^{p}$. But in fact, $\gamma$ has a minimal polynomial which divides $1+x+\cdots+x^{p-1}$, since

$$
b^{1+\gamma+\cdots+\gamma^{p-1}}=1,
$$

for all $b$ in $P$. Thus we have a contradiction, and so $Q$ is normal in $H$, and we are done.

Now we must consider the case that if $B(\neq 1)$ is any $\gamma$-invariant subgroup of $P$, normal in $P$, then $N=N_{H}(B)$ is never equal to $H$. Hence $N$, being $\gamma$-invariant, is an $H_{p}$-group or is a $p$-group, so $N=P_{1} K_{1}$, where $P_{1}$ is a Sylow $p$-group of $N, K_{1}$ is normal in $N$ and is nilpotent, and $K_{1} \cap P_{1}=1$. Since $B$ is normal in $N, K_{1}$ is contained in $C_{N}(B)$, and thus contained in $C_{H}(B)$, so $N_{H}(B) / C_{H}(B)$ is a $p$-group (i.e., is isomorphic to $P_{1} / P_{0}$, for some subgroup $P_{0}$ of $\left.P_{1}\right)$. But then, since this holds for all such $B$, Thompson's theorem ([6]) asserts that $P$ has a normal complement $K$ in $H$; i.e., $H=P K$, where $P \cap K=1$ and $K$ is normal in $H$. Since $K$ consists exactly of the elements of $H$ whose order is prime to $p, K$ is characteristic. Thus $K$ is an $H_{p}$-group (even a Frobenius group) and is nilpotent, so we are done.

\section{BIBLIOGRAPHY}

1. Reinhold Baer, Classes of finite groups and their properties, Illinois J. Math., 1 (1957), 115-187.

2. W. Burnside, Theory of Groups of Finite Order, New York, 1955.

3. P. Hall, A contribution to the theory of groups of prime-power order, Proc. London Math. Soc., 36 (1933), 29-95.

4. P. Hall and G. Higman, On the p-length of p-soluble groups and reduction theorem for Burnside's problem, Proc. London Math. Soc., 6 (1956), 1-42.

5. E. G. Straus and G. Szekeres, On a problem of D. R. Hughes, Proc. Amer. Math. Soc., 9 (1958), 157-158.

6. J. G. Thompson, Finite groups with fixed-point-free automorphisms of prime order, Math. Z. (to appear).

UNIVERSITY OF CHICAGo

De Paul University 



\section{PACIFIC JOURNAL OF MATHEMATICS}

EDITORS

David Gilbarg

Stanford University

Stanford, California

R. A. Beaumont

University of Washington

Seattle 5 , Washington
A. L. Whiteman

University of Southern California

Los Angeles 7, California

L. J. PAIGE

University of California

Los Angeles 24, California

\section{ASSOCIATE EDITORS}

\author{
E. F. BECKENBACH \\ C. E. BURGESS \\ E. HEWITT \\ A. HORN
}

\author{
V. GANAPATHY IYER \\ R. D. JAMES \\ M. S. KNEBELMAN \\ L. NACHBIN
}
I. NIVEN
E. G. STRAUS
T. G. OSTROM
G. SZEKERES
H. L. ROYDEN
F. WOLF
M. M. SCHIFFER
K. YOSIDA

\section{SUPPORTING INSTITUTIONS}

\author{
UNIVERSITY OF BRITISH COLUMBIA \\ CALIFORNIA INSTITUTE OF TECHNOLOGY \\ UNIVERSITY OF CALIFORNIA \\ MONTANA STATE UNIVERSITY \\ UNIVERSITY OF NEVADA \\ OREGON STATE COLLEGE \\ UNIVERSITY OF OREGON \\ OSAKA UNIVERSITY \\ UNIVERSITY OF SOUTHERN CALIFORNIA
}

\author{
STANFORD UNIVERSITY \\ UNIVERSITY OF TOKYO \\ UNIVERSITY OF UTAH \\ WASHINGTON STATE COLLEGE \\ UNIVERSITY OF WASHINGTON \\ $* \quad * \quad *$ \\ AMERICAN MATHEMATICAL SOCIETY \\ CALIFORNIA RESEARCH CORPORATION \\ HUGHES AIRCRAFT COMPANY \\ SPACE TECHNOLOGY LABORATORIES
}

Mathematical papers intended for publication in the Pacific Journal of Mathematics should be typewritten (double spaced), and the author should keep a complete copy. Manuscripts may be sent to any one of the four editors. All other communications to the editors should be addressed to the managing editor, L. J. Paige at the University of California, Los Angeles 24, California.

50 reprints per author of each article are furnished free of charge; additional copies may be obtained at cost in multiples of 50 .

The Pacific Journal of Mathematics is published quarterly, in March, June, September, and December. The price per volume (4 numbers) is $\$ 12.00$; single issues, $\$ 3.50$. Back numbers are available. Special price to individual faculty members of supporting institutions and to individual members of the American Mathematical Society: $\$ 4.00$ per volume; single issues, $\$ 1.25$.

Subscriptions, orders for back numbers, and changes of address should be sent to Pacific Journal of Mathematics, 2120 Oxford Street, Berkeley 4, California.

Printed at Kokusai Bunken Insatsusha (International Academic Printing Co., Ltd.), No. 6, 2-chome, Fujimi-cho, Chiyoda-ku, Tokyo, Japan.

PUBLISHED BY PACIFIC JOURNAL OF MATHEMATICS, A NON-PROFIT CORPORATION

The Supporting Institutions listed above contribute to the cost of publication of this Journal, but they are not owners or publishers and have no responsibility for its content or policies. 


\section{Pacific Journal of Mathematics}

\section{Vol. 9, No. 4 \\ August, 1959}

Frank Herbert Brownell, III, A note on Kato's uniqueness criterion for

Schrödinger operator self-adjoint extensions ............... 953

Edmond Darrell Cashwell and C. J. Everett, The ring of number-theoretic

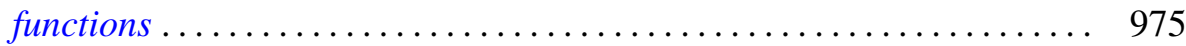

Heinz Otto Cordes, On continuation of boundary values for partial

differential operators ............................. 987

Philip C. Curtis, Jr., n-parameter families and best approximation . . . . . . 1013

Uri Fixman, Problems in spectral operators . . . . . . . . . . . . . . . 1029

I. S. Gál, Uniformizable spaces with a unique structure .............. 1053

John Mitchell Gary, Higher dimensional cyclic elements ............ 1061

Richard P. Gosselin, On Diophantine approximation and trigonometric

polynomials ..................................... 1071

Gilbert Helmberg, Generating sets of elements in compact groups ........ 1083

Daniel R. Hughes and John Griggs Thompson, The H-problem and the

structure of $H$-groups .................................. 1097

James Patrick Jans, Projective injective modules ................. 1103

Samuel Karlin and James L. McGregor, Coincidence properties of birth and

death processes ..................................... 1109

Samuel Karlin and James L. McGregor, Coincidence probabilities ........ 1141

J. L. Kelley, Measures on Boolean algebras ................... 1165

John G. Kemeny, Generalized random variables ................... 1179

Donald G. Malm, Concerning the cohomology ring of a sphere bundle ... . . 1191

Marvin David Marcus and Benjamin Nelson Moyls, Transformations on

tensor product spaces .................................. 1215

Charles Alan McCarthy, The nilpotent part of a spectral operator ........ 1223

Kotaro Oikawa, On a criterion for the weakness of an ideal boundary

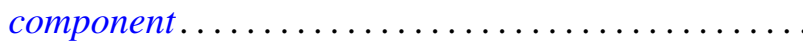

Barrett O'Neill, An algebraic criterion for immersion . . ............... 1239

Murray Harold Protter, Vibration of a nonhomogeneous membrane ... . . . . 1249

Victor Lenard Shapiro, Intrinsic operators in three-space . . . . . . . . . . . 1257

Morgan Ward, Tests for primality based on Sylvester's cyclotomic

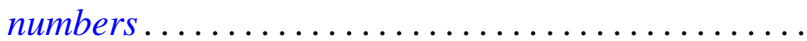

L. E. Ward, A fixed point theorem for chained spaces ....

Alfred B. Willcox, Šilov type $C$ algebras over a connected locally compact

abelian group..................................... 1279

Jacob Feldman, Correction to "Equivalence and perpendicularity of

Gaussian processes" ........................ 